\title{
L'iconologie nostalgique chez R. W. Fassbinder, ou le cinéma comme outil pour une autre histoire de l'art
}

\author{
Jean-Michel DURAFOUR \\ paru initialement dans Nouvelle Revue d'Esthétique, $n^{\circ} 17,2016$, \\ pp. 89-103
}

\begin{abstract}
«Tout ce qui suit participe autant que possible (on ne peut pas abandonner complètement un crustacé quotidien de cinquante ans) de cette respiration de l'éponge où vont et viennent perpétuellement les poissons du souvenir, des alliances foudroyantes de temps, d'états et de matières que le sérieux, ce monsieur trop écouté, trouverait inconciliables. »
\end{abstract}

Julio Cortázar

Le présent texte s'attache à un mode précis de regard iconologique que je nomme pour l'occasion l'iconologie nostalgique. Elle revient à une certaine manière de faire retour (nóstos) sur des œuvres consacrées de l'histoire de l'art, pour l'occasion seulement picturales, en économie cinématographique - le film officiant tout à la fois comme révélateur iconique d'invention pour les figures peintes et instrument méthodologique d'inventaire pour l'histoire de la peinture, en proposant dans ces deux domaines, histoire de l'art et interprétation des figures composées, d'autres pistes que celles que les canons doxiques auront souvent pu retenir. Ici, pourtant, sans regret ni mal-être ; cependant non sans une certaine douleur (álgos) mais exercée sur les ouvres d'art: torsions, plissures, fibromes. On verra comment exactement.

Ce retour particulier, dont le regard se voudrait le commensal, c'est celui qui consiste à y revenir pour voir pour la première fois. Une telle formulation peut paraître au premier abord étrange, voire déraisonnable, le retour semblant impliquer constitutivement la répétition. Il s'agirait donc d'un retour qui ne répète pas, qui ne revient pas. Mais n'est-ce pas la règle ? On revient dans le retour, par le retour, mais le retour lui-même ne revient pas. Le retour vient. Ainsi les références picturales auxquelles je vais m'intéresser dans un instant (sans reproductions, je m'en excuse, mais elles sont très fameuses), ne reviennent pas aux œuvres d'origine, au sens on l'on peut dire que ça revient au même, mais ce sont les æuvres qui reviennent sous une forme d'une inédite actualisation. Je m'attacherai 
à des œuvres picturales en tant qu'elles se présentent, dans et par des films, à nouveau; non de nouveau.

On peut avancer de la nostalgie ainsi conçue, ceteris paribus, ce que Jacques Derrida a bien vu dans le revenant. On commencera par se contenter, peut-être, de cette analogie. Dans un texte consacré à la théorie derridienne des fantômes et à L'Aventure de $M^{\text {me }}$ Muir/The Ghost and Mrs. Muir (1947) de Joseph Mankiewicz, j'écrivais la chose suivante (on me pardonnera de me citer moi-même impoliment mais c'est pour aller directement à ce qui est ici l'essentiel) : «L'événement, c'est ce qui survient, ce qui surgit de (ex/venire); et ce qui vient, c'est toujours ce qui revient : revenir ne veut pas dire autre chose qu'un venir-une-secondefois (par préfixe : re/venire) revenant à un venir-pour-la-première-fois (on décompose alors autrement : res/venire - de res : la chose ; venir à la chose, venir dans le monde) : "Répétition et première fois, voilà peut-être la question de l'événement comme question du fantôme." Le fantôme, ni vivant ni mort, ni hallucination ni perception (ce qui hante : ce qui est entre), ne revient jamais, ne bégaie ni ne radote rien, il est un événement premier du mort, sans antécédent. Le mort peut bien revenir, mais le revenant, lui, ne revient pas : il vient. Il vient d'un revenir qui revient à un venir ${ }^{l}$. " Il en va en quelque sorte de même pour la nostalgie visuelle qui m'occupera : admettons qu'elle vienne d'un revenir qui revienne à un venir.

Retourner signifie étymologiquement revenir faire un tour là où l'on aura toujours d'abord été une première fois avant, jadis, à un point de départ ou un temps paroxystique, réels ou imaginaires, qui manquent, qu'on regrette par la pensée (return, retor) : c'est la noèsis qui accomplit le nóstos ${ }^{2}$. Se retourner, c'est se replier, se façonner (tornare) sur son dos. Je propose, au contraire, de déchronologiser le retour, d'en faire un premier tour vers la chose (res), en déclinant une étymologie tangentielle: res/tornare. Dans l'iconologie nostalgique telle que je voudrais l'appréhender ici, la première fois du regard n'est pas jetée dans le passé, mais lancée vers l'avenir. De même le fantôme derridien est une présence sans regrets : «Le spectre n'est pas seulement l'apparition charnelle de l'esprit, [...] sa vie déchue et coupable, c'est aussi l'attente impatiente et nostalgique d'une rédemption, à savoir, encore d'un esprit

\footnotetext{
${ }^{1}$ Jean-Michel Durafour, «Iconoplasmie. Chemin faisant en compagnie de Jacques Derrida et Gene Tierney », Débordements, avril 2015, http://www.debordements.fr/spip.php?article357. La citation de Derrida vient de Spectres de Marx, Paris, Galilée, coll. « La philosophie en effet », 1993, p. 31.

${ }^{2}$ Et inversement, toute pensée authentique est nostalgie. Voir le célèbre fragment de Novalis : «Toute philosophie est proprement nostalgie - aspiration à être partout chez soi » (Le Brouillon général, $\mathrm{n}^{\circ}$ 857, traduit de l'allemand par Olivier Schefer, édition revue et augmentée, Paris, Allia, 2005, p. 231).
} 
[...]. Le fantôme se serait l'esprit différé, la promesse ou le calcul d'un rachat $^{3}$ ». Le film ne fait pas que revenir à un chez-soi du tableau manifeste avant son action (il le fait sans aucun doute aussi : tel tableau est cité parce qu'il a un certain sens préalable), mais contribue surtout à exhiber un soi, qui aura toujours virtuellement été là, mais qui ne s'engage que dans le cadre de son intervention spéciale. On prendra donc la nostalgie usuelle - qui est «l'irréversion qui prive absolument du regard qui régna ${ }^{4} \gg-$ à rebrousse-poil.

Un dernier mot liminaire. Mes exemples de cinéma seront choisis dans le corpus de Rainer Werner Fassbinder. D'autres signatures pourraient tout autant prétendre exemplifier l'iconologie nostalgique. Une précision rapide, à ce sujet : comme à mon habitude, je ne situe nullement ma démarche au niveau d'intentions conscientes non plus que d'un inconscient, de qui que ce soit - les interventions sur un film sont plurielles - dont je ne peux rien savoir, bref : d'une quelconque intériorité (occulte). Je ne travaille qu'avec ce que j'ai sous les yeux, des phénomènes imageants, et ne fais que regarder des images s'entr'exprimer les unes les autres, sans chercher à explorer des pensées, claires ou obscures, de dedans ou de «derrière » la tête ${ }^{5}$, par définition inaccessibles.

\section{Préambule warburgien}

Dans l'histoire de l'art, l'une des modalités les plus fécondes de cette nostalgie tournée vers l'avant a été mise en place par Aby Warburg à travers la théorie du Nachleben. Je n'ai nullement l'intention de présenter consciencieusement cette méthodologie avec laquelle nous sommes désormais mieux familiarisés grâce à l'exceptionnel travail d'édition, en Allemagne, par les représentants de l'école de Hambourg (Bredekamp, Warnke, Settis, Diers, etc.), des Gesammelte Schriften - Studienausgabe, d'abord à l'Akademie Verlag de Berlin, entre 1998 et 2012, puis chez l'éditeur De Gruyter. Je me contenterai de retenir de Warburg ce qui intéressera la suite de ma démonstration : l'impératif du « ressouvenir en avant », pour reprendre la belle formule de Kierkegaard (La Reprise).

\footnotetext{
${ }^{3}$ Derrida, Spectres de Marx, op. cit., p. 217. Je souligne.

${ }^{4}$ Pascal Quignard, Abîmes. Dernier Royaume III, Paris, Grasset \& Fasquelle, 2002, p. 42.

${ }^{5} \mathrm{La}$ formule est pascalienne. Pour la théorie personnelle sous-tendant une telle méthodologie, je me permets de renvoyer à Brian De Palma. Épanchements : sang, perception, théorie, Paris, L'Harmattan, coll. «Esthétiques», 2013. Voir aussi s'agissant de Souriau plus loin.
} 
La question initialement rencontrée par l'historien de l'art Aby Warburg, dès sa thèse de doctorat sur Botticelli en 1893, est relativement bien connue : "Que signifie l'influence de l'Antiquité pour la civilisation artistique des débuts de la Renaissance ${ }^{6}$ ?» - et cela, plus spécifiquement à propos de l'intensification extérieure du mouvement par ce que Warburg nomme Pathosformeln, des « formules d'expressivité ». L'art antique se caractérise par «la formation d'un style capable de représenter les mimiques expressives de la vie en les exagérant ${ }^{7}{ }^{7}$. Ainsi, rompant avec l'art réaliste du Quattrocento, la Renaissance, d'abord en sculpture puis en peinture, a selon Warburg imposé un retour au style pathétique d'une Antiquité orgiaque et dionysiaque (par référence à Nietzsche; contre la quiétude olympienne de la conception winckelmanienne de l'art antique, alors dominante dans l'histoire de l'art, et son regressus ad antiquitatem).

Les «dynamogrammes de l'art antique » font retour à la Renaissance, malgré la volonté de répression de l'Église catholique, transmis et transformés, quand bien même leur signification initiale a disparu, en « un renversement radical (inversion) de la signification qu'ils avaient pour l'Antiquité classique ${ }^{8} »$. Par exemple - il est donné par Warburg dans Le Rituel du serpent - la pose du père combattant les serpents dans le groupe de marbre du Laocoon est reprise par Galeazzo Mondella dans une plaquette en bronze pour figurer le corps fouetté du Christ pendant sa flagellation. Pour représenter en une image unique et statique des actions vivantes étendues dans le temps et l'espace, les artistes de l'Antiquité en ont retenu les gestes les plus pathétiques; ces gestes sont ensuite devenus si typiques et si puissants qu'ils ont pu rester vivaces en appropriant et agrégeant les sens et les contenus les plus divergents, voire les plus antipodaux. S'en dégagent $1 /$ une « iconologie de l'intervalle ${ }^{9}$ » reposant sur une démarche anthropologique évolutionniste - autre filigrane darwinien (avec la théorie de l'expression de L'Expression des émotions chez l'homme et les animaux de 1872) - visant à rendre possible une histoire relationnelle des formes d'expressions visuelles de l'esprit, et 2/

\footnotetext{
${ }^{6}$ Aby Warburg, Essais florentins, « Art italien et astrologie internationale au Palazzo di Schifanoia à Ferrare », traduit de l'allemand par Sibylle Müller, Paris, Klincksieck, coll. « L'Esprit et les formes », 1990, p. 199.

${ }^{7}$ Ibid., « Albert Dürer et l'Antiquité italienne », p. 163.

${ }^{8}$ Pour les deux citations : Warburg, Allgemeine Ideen, 20, note du 26 mai 1927, cité in Ernst Gombrich, Aby Warburg. Une biographie intellectuelle, traduit de l'anglais (Allemagne) par Lucien d'Azay, Paris, Klincksieck, 2015, p. 235.

${ }^{9}$ Aby Warburg, Miroirs de faille. À Rome avec Giordano Bruno et Édouard Manet, 1928-29 (Écrits I), " Journal romain », 14 avril 1929, établi par Maurizio Ghelardi et traduit de l'allemand par Sacha Zilberfarb, Paris, Les presses du réel/L'Écarquillé, 2011, p. 113.
} 
une « iconologie critique exige[ant] que l'on écarte sans cesse des couches inattendues d'apports incompréhensibles ${ }^{10} »$.

Pour Warburg, l'histoire de l'art est une archéologie métamorphique des formes stylistiques et une Kulturwissenschaft (sans disjoindre l'histoire de l'art et celle de la culture : contrairement à ses contemporains Riegl et Wölfflin), régies par le schème scalaire de la « survivance [Nachleben] » (une transposition du survival de l'ethnologue anglais Edward Burnett Tylor, passé par Julius von Schlosser) des Pathosformeln, rémanence fantomale dans la différence, associé au concept d'origine naturaliste de Leitfossil (Goethe, A. G. Werner, Freud).

\section{Le coup de Poussin}

Warburg pense les formules d'expressivité antiques chargées d'une signification inédite (à la différence de la nostalgie winckelmanienne), il ne s'attache pas aux invariances mais aux procédures de ruptures: perduration de la forme, mais variation du contenu et du style. Je propose d'en prolonger autrement la démarche et d'identifier dans quelques films des présentations d'œuvres d'art caractéristiques, qui par leur intégration constitutionnelle dans une autre image (photographique, mobile), par montage, cadrage, etc., en dégage un sens déjà contenu virtuellement dans l'ancien mais qui ne se fait voir, qui ne s'actualise pour la première fois que par le truchement de certaines quantités de déplacement cinématographique d'énergie (c'est la torsion douloureuse du paragraphe introductif). Il s'agit d'une interprétation iconologique particulière qui, non seulement ne tient aucun compte de la "personnalité de l'artiste » (Panofsky), mais surtout ne porte pas sur le contenu de l'œuvre présente, le film, c'est-à-dire revient, à partir d'actes de description iconographique, sur celui du tableau cité par copie, par transposition, donc par altération dans le film, toujours absent tel quel à notre regard.

Je paie ma dette, ici, à la philosophie d'Étienne Souriau qui a théorisé les modes d'existence virtuelle des objets. "L'artiste, en de tels cas, a charge d'âme vis-à-vis des êtres qui n'en ont pas encore, d'âme, qui n'ont que la simple et plate existence physique. Il découvre ce qui manquait encore à cette chose en ce sens. L'accomplissement qu'il lui confère, c'est bien l'accomplissement authentique d'un être qui n'occupait pour ainsi dire que la place à lui dévolue dans le mode d'existence physique, mais qui restait encore pauvre à faire dans d'autres modes d'existence. Si bien que si cette table physiquement est faite, elle est encore à faire, en ce

\footnotetext{
${ }^{10}$ Warburg, Essais florentins, «Art italien et astrologie internationale au Palazzo di Schifanoia à Ferrare », op. cit., p. 295.
} 
qui concerne l'artiste ou le philosophe ${ }^{11}$. » Évidemment, un tableau n'est pas comme une table, il n'est pas artistiquement à faire, il l'est déjà (à moins de supposer une lecture variante de la proposition de Marcel Duchamp : «Se servir d'un Rembrandt comme planche à repasser »...), mais il n'est pas impossible d'infléchir légèrement le texte sourialien, mieux : de le prolonger entre les modes d'expression artistique. Ainsi, un tableau, bien que peint des siècles avant l'émergence du cinéma, pourrait voir, en tant qu'objet pictural de la prise de vue photographique, son sens exposé augmenter par sa prise en charge filmique, en glissant la représentation d'un type de procédés artistiques à un autre.

Je prends un premier exemple, très bien connu, sur lequel on a déjà beaucoup écrit, peut-être trop, dans Les Larmes amères de Petra von Kant/Die bitteren Tränen der Petra von Kant (1972) : le Midas devant Bacchus (1629-1630) de Poussin, mais dont je vais proposer une lecture nostalgique rafraîchissante.

1/ Il s'agit d'une reproduction en papier peint sur tout un mur, agrandie et sans cadre, occupant tout le mur du fond de la scénographie. Ainsi, les figures sont étirées jusqu'à une taille supérieure à celle de n'importe quel être humain, notamment ceux qui évoluent à tour de rôle devant elles, dans la chambre à coucher de Petra, et il est aisé d'y repérer une mise en abyme de la projection cinématographique sur grand écran. La taille et l'absence de bordure favorisent l'impression que l'image suinte, se prolonge au-delà, dans le milieu environnant. Cette impression est, elle aussi, proprement cinématographique et peut se voir comme une mise en image indirecte - par la peinture -, c'est-à-dire théorique, du hors-champ. L'effet est d'autant plus habile que, comme l'écrit Stanley Cavell, «le monde d'une peinture n'est pas en continuité avec le monde de son cadre ; à son cadre, le monde trouve ses limites. Nous pourrions dire : une peinture est un monde ; une photographie est photographie $d u$ monde $^{12} »$. C'est que la peinture figurative - puisqu'elle seule m'occupe ici - n'a pas de hors-champ. Commentant la fenêtre albertienne et l'invention de la perspectiva artificialis, Daniel Arasse précise : "La première opération du peintre, avant le point de fuite, c'est ce qu'on appellerait aujourd'hui le cadrage, c'est-à-dire le fait de poser le cadre à l'intérieur duquel on pourra contempler l'histoire. Je le répète parce que j'y tiens beaucoup, la fenêtre d'Alberti n'ouvre pas du tout sur le monde, ce n'est pas un détail du monde qu'on voit à travers cette fenêtre, c'est le cadre à partir duquel on peut contempler l'histoire ${ }^{13}$.» Victor Stoichita le dit autrement:

\footnotetext{
${ }^{11}$ Étienne Souriau, Les Différents Modes d'existence suivi de Du mode d'existence de l'œuvre à faire, Paris, PUF, coll. « MétaphysiqueS », 2009, p. 198.

12 Stanley Cavell, La Projection du monde, Paris, Belin, 1972, p. 51.

${ }^{13}$ Daniel Arasse, Histoires de peinture, Paris, Gallimard, coll. « Folio Essais », 2006, p. 87. Le hors-champ, rappelle quant à lui Hubert Damisch, n'est présent dans la
} 
«L'espace de l'image est visuellement "perceptible", sans être physiquement "praticable"14. $\gg$ Avoir choisi Poussin est, de la part de Fassbinder, d'autant plus ingénieux que ce peintre est spécialement connu pour le soin qu'il accordait aux choix de ses cadres, comme il l'indique à Félibien dans une lettre du 26 février 1648 ou à l'ami Chantelou, à qui il vient d'envoyer Les Israélites recueillant la manne dans le désert, dans une autre lettre antérieure, non moins célèbre, du 28 avril 1639. Le cadre est la condition de visibilité du tableau ${ }^{15}$. (Le générique qui fait attendre la caméra au seuil de la chambre, et du film, avant d'entrer, cadre des chats sur les marches d'un escalier; du bois sont les faits les cadres. La tradition des figures de bord donnant illusoirement l'impression d'enjamber l'espace fictionnel de la toile et l'espace réel de l'observateur, voire d'appartenir à ce dernier espace uniquement, comme posées sur la toile physique, plutôt que d'appartenir à la représentation, pour notamment souligner la présence $d u$ cadre, est tout spécialement animalière, il est vrai sur des animaux de petites tailles: mouches, sauterelles, escargots...)

2/ Pour un cinéaste post-brechtien comme Fassbinder, la question des limites de la représentation est évidemment sensible à un autre niveau : rendre visibles les artifices de l'image et de la mise en scène. Le choix d'un peintre français comme Poussin est aussi une manière oblique de renvoyer à la théorie du quatrième mur chère à Diderot. (Diderot avait déjà - en guise de transition avec le point d'avant - proposé aux visiteurs des salons de regarder les tableaux à travers des miroirs éliminant leur cadre pour en estomper la césure avec le monde : faut-il l'inscrire dans l'histoire qui conduira au cinéma $\left.?^{16}\right)$. Le quatrième mur est la paroi

peinture que s'il est convoqué, par un effet de miroir ou autre (Un souvenir d'enfance par Piero della Francesca, Paris, Le Seuil, coll. «Librairie du XXI ${ }^{\mathrm{e}}$ siècle», 1997, p. 130).

${ }^{14}$ Victor I. Stoichita, L'Effet Sherlock Holmes. Variations du regard de Manet à Hitchcock, Paris, Hazan, 2015, p. 14.

${ }^{15}$ Commentant la lettre à Chantelou, Louis Marin signale : «Le cadre est l'ornement du tableau, mais c'est un ornement nécessaire : c'est une des conditions de possibilité de la contemplation du tableau, de sa lecture et, par là, de son interprétation. [...] Avec le cadre, le tableau inscrit en lui-même sa propre théorie, c'est-à-dire le fait de se présenter théoriquement pour représenter quelque chose» (De la représentation, " Figures de la réception dans la représentation moderne de peinture », établi par Daniel Arasse, Paris, Gallimard/Le Seuil, coll. « Hautes Études », 1994, pp. 316-317). ${ }^{16} C f$. la notoire théorie de Michael Fried d'inspiration diderotienne de l'absorbement, ici non pas de la figure dans sa vie intérieure à l'exclusion du spectateur (peinture dramatique : Greuze, Van Loo), mais du spectateur dans l'environnement de la toile (peinture pastorale: Vernet) : La Place du spectateur. Esthétique et origines de la peinture moderne, traduit de l'anglais (États-Unis) par Claire Brunet, Paris, Gallimard, coll. « Nrf-essais », 1980. 
imaginaire séparant l'action scénique de l'espace de la salle afin d'atténuer au mieux l'artifice du dispositif. En économie représentationnelle classique, ce mur est univoquement transparent : nous voyons les acteurs, mais les acteurs ne sont pas censés nous voir. Le mur est virtuel pour moi ; pour l'acteur réel. La scène ne s'adresse pas au spectateur. De la poésie dramatique: «Soit donc que vous composiez, soit que vous jouiez, ne pensez non plus au spectateur que s'il n'existait pas. Imaginez sur le bord du théâtre un grand mur qui vous sépare du parterre : jouez comme si la toile ne se levait pas. » Toile : pour Diderot, le théâtre est une succession de tableaux - «une disposition de ces personnages sur la scène, si naturelle et si vraie, que rendue fidèlement par un peintre, elle me plairait sur la toile »-et, inversement, la peinture est un théâtre muet (Lettre sur les sourds et muets). Ces problématiques sont d'autant plus sensibles dans la cocotte-minute théâtralisante (unité de temps, de lieu, d'action; division en actes) des Larmes amères de Petra von Kant, adapté d'une pièce de Fassbinder écrite un an auparavant.

$3 /$ Le tableau représente très exactement le moment où le roi phrygien Midas, à genoux, pénitent, sur la droite, remercie Bacchus, à gauche et debout, de l'avoir délivré du don empoisonné (il ne peut plus manger) de transformer tout ce qu'il touche en or. Le sens du tableau est ambigu car on pourrait y lire également le moment où Bacchus accorde son don à Midas en échange de son hospitalité à l'égard du vieux Silène, qu'on peut voir ivre sur la gauche, tout près du dieu. Devant les trois personnages, au premier plan, une bacchante est endormie au pied du satyre, avec un garçonnet, une cruche de vin et un bol renversés à ses pieds, tandis que deux autres jouent avec un bouc traditionnel sur la droite (Bacchus enfant a été transformé en chevreau par Mercure, après avoir été découpé et bouilli par les Titans, pour le confier aux nymphes de Nysa). Derrière Bacchus, au troisième plan, dans un petit bosquet, on peut voir trois suivants (des bergers sans doute) : l'un cueille des grappes de raisin, un autre joue d'un aulos devant le dernier qui écoute assis par terre. Dans le fond, plus dégagé et ouvert derrière sur l'horizon (la ligne de crête des montagnes), deux hommes contre un rocher sont également amollis. L'ensemble baigne dans une tranquillité grise. - On a donc affaire à un tableau à deux sens compossibles. Poussin a, par ailleurs, beaucoup représenté la figure de Bacchus. Elle apparaît dans de nombreux tableaux: Bacchus et Ariane (1625-1626), l'Enfance de Bacchus (c. 1626), deux Nourriture de Bacchus (c. 1626-1627), Bacchus-Apollon (c. 1626-1628), Le Triomphe de Pan (Bacchanales Richelieu III) et Le Triomphe de Bacchus (Bacchanales Richelieu IV) (1635-1636), La Naissance de Bacchus (1657) - sans compter toutes les bacchanales plus ou moins indirectement raccordées au mythe dionysiaque: deux Bacchanale d'enfants (1626), Scène bachique ou Nymphe chevauchant 
un satyre (1626), Bacchanale à la joueuse de guitare ou Grande Bacchanale (1627-1628), Scène bachique ou Nymphe et satyre buvant (1626-1628), Bacchanale devant un terme de Pan (1637-1638), Le Triomphe de Neptune (Bacchanales Richelieu I) (1635-1636), Le Triomphe de Silène (Bacchanales Richelieu II) (1637). Poussin a peint plus de Bacchus que de Christs. Quant à Midas, Poussin l'a également figuré deux fois dans les années précédentes: Midas à la source du Pactole et Midas se lavant dans le Pactole (c. 1626-1628). Le choix de Midas devant Bacchus a sans doute à voir avec le souci d'indiquer quelque chose à propos de la peinture de Poussin en général, en même temps qu'il offre le cas d'une œuvre déjà préalablement, et indépendamment de l'intervention du film, ouverte à la polysémie.

À partir de là, comment le tableau de Poussin fonctionne-t-il plastiquement, figurativement dans le film? J'en rappelle d'abord l'argument rapidement. Petra (Margit Carstensen), créatrice de mode, tombe amoureuse de Karin (Hanna Schygulla), une jeune ouvrière qu'elle héberge et dont elle décide de faire son mannequin attitré, au grand dam de sa secrétaire, Marlene (Irm Hermann), également éprise de sa patronne et qui ne mène plus, par amour, qu'une existence ancillaire et humiliée. La jalousie de Marlene dévore le trio, jusqu'à ce que Petra tombe elle aussi dans la servitude amoureuse quand Karin annonce qu'elle retourne auprès de son mari... Le film, tourné dans un décor unique, l'appartement de Petra, se présente comme un huis-clos étouffant, strictement féminin, et pris, comme presque toujours chez Fassbinder, du moins à partir de 1971, entre le kitsch trivial et «baroquisant» du mélodrame sous influence sirkienne (selon les films : dorures, miroirs, spirales, gazes, scintillements, outrance des fards, objets floutés en amorce, métachromasie, poupées, etc. - tout n'est qu'images, et images d'images) ${ }^{17}$, et une perception inégalée du pathétique, de la cruauté des affects, de la hideur des chairs, de l'épuisement des êtres, en regard de quoi le moelleux propre à la touche poussinienne, par quoi le peintre entendait résoudre la querelle du coloris et $\mathrm{du}$ dessin ${ }^{18}$, constitue un contrepoint détonant et détonnant.

\footnotetext{
${ }^{17}$ Douglas Sirk (1897-1987), cinéaste d'origine allemand (Hans Detlef Sierck) exilé aux États-Unis en 1937, maître du mélodrame hollywoodien lacrymal et chromatique des années 1950, lui aussi toujours au bord de la catastrophe : Le Secret magnifique/The Magnificent Obsession (1954), Tout ce que le ciel permet/All That Heaven Allows (1955), Mirage de la vie/Imitation of Life (1959). La découverte de six de ses films en 1971, lors d'une rétrospective munichoise, a eu un impact décisif sur l'esthétique de Fassbinder.

${ }^{18}$ Félibien écrit : «J'ai su du Poussin même combien il estimait la couleur du Titien, la beauté et le moelleux de son pinceau: on ne peut nier que dans les premiers ouvrages qu'il fit à Rome il n'ait vraiment imité les coloris de ce savant Vénitien ; on peut cependant remarquer qu'à mesure qu'il se perfectionnait il s'attachait de
} 
Ce dialogue visuel entre le tableau de Poussin et la fable cinématographique est souligné par la mise en scène du film et en place des corps : Midas devant Bacchus offre, tout au long des cadrages et du montage, un modèle de poses et de compositions de figures imitées par les personnages du récit. Ainsi de nombreux plans se donnent à voir comme des sortes de «tableaux vivants» du tableau, chacune des femmes prenant tour à tour telle ou telle attitude, de Bacchus, de Midas, de la bacchante au pied du dieu, voire de Silène ${ }^{19}$.

Cette nostalgie iconologique, ce soin de revenir y voir, dont les effets nous échappent encore, est exhibée dès l'ouverture du film. Petit matin. En un lent mouvement d'appareil vers la droite, le premier plan rase le sol, à hauteur d'homme accroupi, de l'entrée de la chambre, et de la bacchante de la copie de Midas devant Bacchus, jusqu'au lit de Petra, à l'autre bout du trajet, où celle-ci s'éveille. Ce réveil renvoie iconiquement, dans le drapé, le mouvement des bras, la couleur des cheveux, le sommeil, à la posture de la femme assoupie sur le sol du tableau : mais comme en reflet de miroir, tout ce qui est à gauche - bras étiré, port de la tête - passant à droite, et inversement (ce procédé de symétrie spéculaire est cher à Fassbinder). Nous sommes ici pleinement, en première intention, dans le Nachleben de Pathosformeln en économie de création figurative par surcroît, par extensions, telle qu'abordée avec l'orthodoxie warburgienne tout à l'heure. Et ce, à double titre : d'abord parce que les objets de cette survivance sont des formules d'expressivité excessive de la vie en mouvement; ensuite parce que la signification, et pas seulement l'orientation formelle, des formules en est radicalement renversée : par exemple, les bacchantes sont réputées vierges, tandis que Petra est à la fois veuve, divorcée et lesbienne. On notera également deux régimes admirables de détails contribuant à tresser l'un avec l'autre espace fictionnel (le mythème grec) et espace réel (la chambre) plus efficacement, plus souplement, en économie continuée, pour une meilleure circulation des gestes et «dynamogrammes ». L'un est matériel (filmé) ; l'autre, formel (filmant).

préférence aux belles formes et à la correction du dessin »(Vie $d u$ Poussin). Cela ne veut absolument pas dire que Poussin ait abandonné le moelleux en s'intéressant plus au dessin avec l'âge ; bien au contraire. Le peintre anglais Reynolds note, quant à lui : «Le Poussin, dans les derniers temps de sa vie, changea sa manière sèche contre une plus moelleuse et plus riche, où l'on remarque une plus grande liaison entre les figures et le terrain » (cité in Marie Graham, Mémoire sur la vie de Nicolas Poussin, Paris, chez Pierre Dufart, 1821, pp. 110-111; je souligne).

${ }^{19}$ Sur le tableau vivant, je me permets de renvoyer le lecteur francophone à Horst Bredekamp, Théorie de l'acte d'image, traduit de l'allemand par Frédéric Joly en collaboration avec Yves Sintomer, Paris, La Découverte, coll. "Politique et sociétés », 2015, pp. 93-113. 
Le premier: l'interrupteur, au-dessus de la bacchante, avec effet illusionniste d'appartenance à la représentation picturale, près de son bras - comme si c'était elle qui allait l'actionner (la lumière se fera très vite, mais autrement : Marlene va ouvrir la persienne), et inscrivant dès la composition poussinienne sa rémanence zombi dans un environnement tardif, au sein d'une culture, technologiquement, idéologiquement, etc., tout autre (la République fédérale allemande). Cet interrupteur ressemble, problématisant l'intersection et l'articulation entre un signifiant et un signifié, à ce que Jacques Lacan a nommé point de capiton $^{20}$, du nom des boutons piqués à la surface d'un tissu matelassé et nécessaires à la tenu du rembourrage, et donc à la teneur de la chose dans l'entrelacs mixte de son intérieur, de sa profondeur, de son épaisseur avec sa face externe. On trouverait un même effet de perméabilité topologique en raccord interne, marqué plus volontiers par l'angle de la prise de vue, et donc à cheval avec le second exemple que je vais examiner tantôt, à savoir la forme $d u$ film, avec le bol peint déversant son " contenu » sur le tapis : non pas le vin attendu (une tache, par exemple), mais une insolite chute de tissu Petra est modiste.

Le second : le travelling latéral qui balaie la scène de gauche à droite, et relie la bacchante à gauche (du tableau de Poussin) à Petra à droite (du plan de Fassbinder), en un seul environnement homogène et transmédiatique, annulant la fracture, dans le récit filmique, entre la fiction et le réel. La bacchante commence par être aussi à gauche dans le plan; et ne voudrions-nous pas dès lors compléter ce carré cardinal comme suit : Petra est à droite dans le tableau de Poussin?

Ou à tout le moins : Petra est dans le tableau. Poussin l'a peinte quelque part dans Midas devant Bacchus. (Avancerais-je peut-être une piste supplémentaire, en jouant sur des mots qui ont des résonnances bien spécifiques, surtout pour les lecteurs mariniens : Kant est dans le tableau de Poussin ${ }^{21}$ ?) Que faut-il entendre par «Petra est dans le tableau de Poussin »? Il ne s'agit évidemment pas de le prendre au pied de la lettre - ce qui n'aurait aucun sens. Je propose de le traduire ainsi : le travelling laisse voir, comme on laisse entendre, que le tableau de Poussin déborde (on l'a vu: pas de cadre, figures agrandies, etc.) dans le monde réel du film, mais aussi inversement que ce monde réel, l'appartement de Petra en l'occurrence, Petra plus précisément même, est déjà présent, s'imborde dans le tableau.

Nous entrons dans la lecture expressément nostalgique : celle qui va faire retour sur le tableau pour y voir pour la première fois ce qui y a

${ }^{20} C f$. Jacques Lacan, Écrits II, « Subversion du sujet et dialectique du désir », Paris, Seuil, coll. « Points Essais », 1999.

${ }^{21} \mathrm{Je}$ fais évidemment référence au titre de l'ouvrage de Louis Marin, Sublime Poussin, Paris, Seuil, coll. « L’Ordre philosophique », 1998. 
toujours été, en acclimatant notre regard par le truchement d'une mutation filmique ${ }^{22}$. Pour cela, il faut déplacer, et le film, un film sans aucun homme, nous y invite, la figure centrale du tableau du couple formé par Midas et Bacchus vers la bacchante, c'est-à-dire la femme à l'image. Fassbinder commence par la filmer; il en fait explicitement, passé le générique, la figure admonitrice d'entrée ${ }^{23}$ dans le film - ce qui l'assimile à Petra: première figure humaine de chair à apparaitre à l'écran - mais aussi dans le tableau, ce qui est plus audacieux. Le film nous incite à voir le tableau ainsi : la bacchante est une survivance expressive à rebours de Petra. C'est précisément cela l'iconologie nostalgique : faire retour sur la peinture pour voir comme on n'avait jamais vu, avec le film comme outil d'expertise. Pourquoi ? En quoi ? Autant de questions auxquelles il nous est impossible de répondre. Mais peut-être tenons-nous là ce qui explique l'incertitude que j'ai évoquée concernant le sens du tableau - Midas réclamant son don ou implorant, au contraire, que son don soit annulé -, à savoir que le tableau, nous l'avons toujours pressenti, n'est pas une illustration d'un récit mythologique, avec bacchante dénudée dans un coin, mais - ce que le peintre dévot, tel $\mathrm{du}$ moins que nous l'a légué le $\mathrm{XIX}^{\mathrm{e}}$ siècle $^{24}$, ne pouvait pas aborder directement, ce qui se peint - un nu fortement érotisé (vase sous l'aisselle, pénis tourné vers le corps, tête de l'enfant aux fesses, bouc lubrique: toute la zone occupée par la bacchante et l'environnement immédiat qu'elle dirige sont glandulaires, érectiles, olfactifs, sur lesquels opère une carnation invasive contaminant toute la composition), dans le cadre d'un prétexte mythologique, peut-être narrativement premier (la fable imagée), mais picturalement secondaire (l'acte imageant) ${ }^{25}$. Avant tout, il faut regarder le lieu de la femme : il constitue le centre vectorisant de la représentation. Cette endormie est d'ailleurs figure hautement problématique puisque Poussin a probablement fusionné en elle une bacchante mais aussi une nymphe, autre type d'accompagnantes, mais secondaires, du cortège dionysiaque (les ménades sont généralement

\footnotetext{
${ }^{22}$ Cette démarche se distingue de celles qui analysent le cinéma comme instrument d'exégèse pour l'histoire de l'art, et qui produisent des résultats tout à fait passionnants. Pour les pistes récemment ouvertes en France sur ces questions, $c f$. notamment la thèse de doctorat de Joséphine Jibokji, « Simulacres cinématographiques : l'art en fiction dans le cinéma français des années 1960 », université Paris-Sorbonne, sous la direction de Pierre Arnauld, soutenue le 4 décembre 2015.

${ }^{23}$ Cf. Leon Battista Alberti, De la peinture/De pictura (1435), traduit du latin par Jean Louis Schefer, paris, Macula/Dédale, coll. « La Littérature artistique », 1999, p. 179.

${ }^{24}$ Rien de tel, en effet, chez les premiers biographes du peintre : Bellori, Sandrart, Félibien.

${ }^{25}$ Et déjà érotisé par la figure de Bacchus : procession phallophore, orgie sexuelle des bacchanales, bouc prolifique, etc.
} 
vêtues, mais l'histoire de l'art témoigne de bacchantes nues). Faire porter le regard d'abord sur elle, comme le propose Les Larmes amères de Petra von Kant, oriente ainsi Midas devant Bacchus vers l'iconographie traditionnelle des Vénus allongée, de plein air (Vénus endormie de Giorgione, Vénus de Palma le Vieux) ou non (Vénus d'Urbin du Titien). La frontalité sexuelle - sadisme, masochisme, saphisme - est, au contraire, au cœur des Larmes amères de Petra von Kant. Il n'y a pas d'hommes dans le film mais le corps nu géant de Bacchus et son sexe en évidence dévorent le champ : les femmes y sont très souvent cadrées le visage juste en dessous ou par-dessus ${ }^{26}$.

Avancer Petra est déjà dans le tableau de Poussin, c'est exactement mettre le doigt sur la dimension érotique du tableau que le film, tel qu'il le cadre, le monte, le met en images, nous invite à le voir. Je n'ai retenu ici qu'un plan. Un seul. Comme je l'ai précisé, le film multiplie les mêmes procédures de mimétisme et d'inscription imaginaire avec Midas devant Bacchus par rapport aux deux autres femmes du récit, Karin et Marlene. Mieux : selon les moments, un même personnage du film peut prolonger la posture de figures très différentes dans le tableau. Tout de suite après le plan dont j'ai parlé, Petra passe un appel au téléphone. Celui-ci lui est apporté par Marlene. Marlene reprend alors la pose de Bacchus tendant la main vers Midas agenouillé, mais la main droite a remplacé la main gauche (le corps du dieu est d'un blanc lumineux, la robe est noire). Petra dans son lit, celle de Midas (même inversion des mains, mais dans l'autre sens : la gauche, qui prend le combiné, remplace la droite avancée par le roi phrygien). C'est désormais à Midas que le film associe Petra. Il n'y aura fallu que quelques plans. Au demeurant, c'était prévisible: j'ai montré précédemment comment le travelling introductif révélait Petra dans la droite du tableau. Donc à la place occupée par Midas. Ce simple échantillon - tout le film en est tissé suffit à donner une idée de la richesse iconologique du recours nostalgique au Midas devant Bacchus par Les Larmes amères de Petra von Kant.

\section{Fornix Fornarina}

Ce trait iconologique nostalgique, d'une inventivité remarquable, et qui fait du cinéma un outil méthodologique pour une histoire de l'art

\footnotetext{
${ }^{26}$ L'association entre sexualité et peinture est récurrente dans le corpus fassbinderien, comme en atteste Despair (1978), exempli gratia, où le désir homosexuel de Hermann s'affirme quand il observe une nature morte tandis que Félix se déshabille: deux roses encadrent une pipe ouvertement phallique; alors que celui sur lequel il tombe en retrouvant sa femme représente deux pommes avec un cendrier vide
} 
différente (Poussin, peintre chrétien et platonicien ${ }^{27}$, comme peintre de nus?), est récurrent dans le cinéma fassbinderien, même si les occurrences de tableaux objectivement mis en scène, et pas simplement utilisés comme des éléments du décor (intérieurs bourgeois), sont relativement rares. Là encore, je n'ai évidemment pas le temps d'entrer dans trop de détails.

J'examinerai juste un autre cas, aux procédures sensiblement différentes. Si Les Larmes amères de Petra von Kant avait attiré le regard sur tel morceau du tableau, en le fragmentant par les procédures filmiques (focalisation, cadrage...), ce nouvel exemple va, par l'attitude d'un personnage, greffer le tableau sur une lignée iconographique différentielle. Il s'agit d'une mention, fugace, de La Fornarina (15181520) de Raphaël, dans Le Rôti de Satan/Satansbraten (1976). Ce film à la fois burlesque et tragique, drôle et malsain, tendre et ordurier, est saturé d'idiots ou de pervers aux comportements tous plus excentriques les uns que les autres. Ainsi Ernst (Volker Spengler), le frère mentalement attardé de Walter Kranz (Kurt Raab) - un poète révolutionnaire en crise d'inspiration qui va peu à peu s'identifier à Stefan George -, passe son temps à pincer les tétons des filles. Lorsqu'Andrée (Margit Carstensen), la nouvelle maitresse de Walter, s'installe dans l'appartement conjugal, Ernst cherche logiquement à faire connaissance avec elle. Mais celle-ci, pour se protéger, met en écran contre sa poitrine une reproduction de $L a$ Fornarina qui traînait étonnamment par là, c'est-à-dire qu'elle lui offre l'image d'une femme en train de cacher son sein.

La Fornarina est l'un des derniers tableaux de Raphaël, d'attribution discutée, probablement inachevé, repris peut-être à la mort du peintre par son élève Giulio Romano : il portrait celle qui a vraisemblablement été la maîtresse du maître d'Urbino durant les dernières années de sa vie. Celleci fut finalement identifiée comme étant Margherita Luti, la fille du boulanger Francesco Luti da Sienna - d'où son surnom en dialecte romain (fornarino). La femme, sourcils cambrés, yeux en dragée, visage ovale, petit menton, front bas, traits timides mais le regard aguicheur, est représentée en buste, assise, affublée d'un turban de style oriental orné d'un bijou, un ruban bleu liseré d'or à son bras gauche (portant la signature du peintre), la poitrine à demi nue. Elle fait le geste de couvrir son sein gauche avec sa main droite. Le peintre l'aurait également prise pour modèle dans La Donna velata (1515), le pendant chaste de La Fornarina, mais le fait est contesté ${ }^{28}$.

${ }^{27}$ Cf. notamment Yves Bonnefoy, Dessin, couleur et lumière, paris, Mercure de France, p. 139.

${ }^{28}$ Cf. notamment Konrad Oberhüber, Raphaël, traduit de l'allemand par Jean-François Alain, Paris, Éditions du Regard, 1999. 
La Fornarina n'est pas peinte le sein recouvert mais en train de le voiler, comme le voulait la continence de l'époque : ainsi, de part et d'autre, on peut penser à La Naissance de Vénus (1484-1486) de Botticelli ou à la Marie-Madeleine (1533) de Titien. On notera la subtilité du geste d'Andrée : elle montre le sein d'une autre, qui n'est pas un vrai sein, mais seulement une illusion, à l'endroit du sien, comme une combinaison, un survêtement de tracés et de pigments, pour soustraire un autre sein, réel, le sien donc, maintenu dans le revers, l'ourlet de la peinture. Mais Ernst (qui est aussi, au passage, un nom de peintre), comme les pigeons de Zeuxis, s'y laisse prendre et agace le sein plat de peinture. S'y trompe-t-il vraiment? Peu importe. Seul compte qu'il en vienne alors à brancher $L a$ Fornarina, à son insu, par revenance d'une certaine "formule d'expressivité » que je vais préciser, sur une autre voie iconographique : celle des tableaux (femmes au bain, scènes grivoises, etc.) à seins pincés ou pelotés, désignons-les ainsi, comme le fameux Gabrielle d'Estrées et une de ses sæurs (1594) de l'école de Fontainebleau ou encore Une béguine et un moine (1591) de Cornelis Cornelisz van Haarlem.

Entendons-nous. Il n'est pas question de dire que Ernst érotise le portrait de Margherita. La dimension érotique est déjà visible dans le portrait original. Dissimuler un sein est une action hautement sensuelle (caresse autoérotique, frottement du tissu sur la peau) : le voile renaissant, d'une manière générale, attise toujours plus le désir (le nombril ici...) qu'il ne tranquillise la pudeur. Dans La Fornarina, Raphaël fixe une excitation voluptueuse à lui adressée, et à laquelle il retourne l'activité de peinture ${ }^{29}$. Selon Vasari, Raphaël était tellement amoureux de cette femme qu'il ne pouvait pas travailler quand il était loin d'elle : «Lorsqu'il peignit pour son cher ami Agostino Chigi la première loggia de son palais [la villa Farnesina], Raphaël ne pouvait se tenir à son travail, à cause de son amour pour sa maîtresse. Agostino en était désolé. Avec des complicités, il s'arrangea non sans peine pour que cette femme vienne vivre chez Raphaël près de l'endroit où il travaillait et l'ouvrage fut terminé ${ }^{30}$. » Impossibilité de peindre en l'absence de Margherita, à tel point que leur relation est devenue «la relation artiste/modèle archétypique de la tradition occidentale ${ }^{31} »:$ la femme est clairement identifiée à la peinture elle-même.

\footnotetext{
${ }^{29}$ Lieu commun : le pinceau (penicillus : littéralement « le petit pénis »), en est un substitut. $C f$. Daniel Arasse, On n'y voit rien, Paris, Gallimard, colL. « Folio essais », 2000 , p. 119.

${ }^{30}$ Giorgio Vasari, Les Vies des meilleurs peintres, sculpteurs et architectes, tome 5, traduit et édité sous la direction d'André Chastel, Paris, Berger-Levrault, coll. « Arts », 1983, p. 216.

${ }^{31}$ Marie Lathers, Bodies of Art. French Literary Realism and the Artist's Model, Lincoln, University of Nebraska Press, 2001, p. 61. Je traduis.
} 
Par ailleurs, si dans La Fornarina, Margherita parait plus laide que dans La Donna velata, comme le remarquait déjà Stendhal (Idées italiennes: «... le nez est trop grand, trop avancé... »), à tel point qu'il est difficile d'avouer y voir le même modèle, Arasse l'interprète ainsi : "La beauté parfaite du corps nu de La Fornarina et le modelé individuel, moins parfait, de son visage incitent à voir, dans cette dimension interne de l'œuvre, l'indice d'une érotisation de la figure. [...] C'est l'Éros d'un désir physique qui n'a rien de platonicien ${ }^{32}$. » C'est précisément cette dimension « ouvertement érotique » qui explique, aux yeux de l'historien de l'art, qu'on trouve à La Fornarina moins de grâce, de venustà (pourtant un attribut distinctif du style raphaélesque) et qu'elle ne plaise pas universellement. La photographe Cindy Sherman s'en souviendra dans Untitled \#205 (1989).

Ingres, ce grand admirateur du peintre de la Madone à la prairie, ne s'y est pas trompé, qui composera en 1814, dans sa période troubadour, une œuvre intitulée Raphaël et la Fornarina, dont il fit plusieurs versions. Il en réalisa également une variante tardive en 1840 . On y assiste à une pause dans l'exécution de La Fornarina: nous sommes dans l'atelier du peintre. L'amante est assise sur les genoux de l'artiste : elle reprend la posture de la Madone à la chaise (v. 1514), pour lequel elle avait déjà servi vraisemblablement de modèle, et que Ingres reproduit partiellement au fond de l'atelier (le peintre a remplacé l'enfant Jésus dans ses bras). Tandis que Raphaël, en contrapposto (corps tourné à gauche, tête vers la droite), regarde son tableau encore inachevé dans l'alignement du bord latéral, la femme n'y jette pas un œil mais toise, au contraire, fermement le spectateur dans l'axe (veduta perspective au fond à gauche), comme si nous avions surpris un moment intime auquel, loin de nous chasser, elle nous invitait coquinement à assister: ils s'enlacent, vont s'embrasser sans doute. Son regard possède la même lumière malicieuse que dans le portrait de Raphaël : sauf qu'ici la Fornarina ne regarde plus son amantce qui était finalement attendu - mais le spectateur, le tiers voyeur: l'effet - il faut connaître le tableau de Raphaël - est volontairement beaucoup plus déstabilisant : notre regard ne vient plus se superposer à celui du peintre exécutant, à savoir s'y dissoudre, se retirer sous le regard du peintre.

Mais allons plus loin. Cette femme, la Fornarina, cache-t-elle son sein ou s'apprête-t-elle plutôt elle-même - c'est ainsi que le geste de Ernst dans Le Rôti de Satan dirige notre regard - à le pincer, à jouir de sa chair érogène ? Nous ne serions plus alors dans un moment seulement érotique mais carrément salace voire obscène. La femme convie son amant à

${ }^{32}$ D. Arasse, «L'atelier de la grâce, in Raphaël. Grâce et beauté, Skira/Musée du Luxembourg, 2001, pp. 66-67. 
l'acte sexuel (main gauche sur son sexe). C'est d'ailleurs quelque chose sur quoi les détracteurs de La Fornarina, très tôt, ont insisté, certes différemment, en soulignant une connotation sexuelle déplaisante dans l'image, mais seulement encore comme d'une trace, d'un vestige non représenté tel quel : La Fornarina serait le portrait d'une prostituée (Charles de Brosses, Giovanni Morelli). En 1837, dans L'État actuel de l'art religieux en France, Charles de Montalembert soutient, dans un vocabulaire qui ne cherche pas à dissimuler son mépris, que Raphaël, en même temps qu'il a porté l'art chrétien à sa perfection, a aussi initié luimême la décadence maniériste qui allait suivre. La responsable? La Fornarina. «... Ce Raphaël dégénéré qui n'a plus pour modèle que sa Fornarina $^{33}$. »

Ingres avait déjà mis le spectateur sur une piste plus sexuelle qu'érotique (Vasari rapporte également que Raphaël est mort d'épuisement sexuel ${ }^{34}$ ). C'est ce caractère concupiscent de La Fornarina que Le Rôti de Satan, multipliant les scènes grivoises, sadomasos (insultes, crachats, meurtres), impudiques (les personnages y font l'amour devant tous les autres), nous invite à y voir avant tout. Et nous y apprenons au passage quelque chose d'important concernant l'iconologie nostalgique: il n'est pas nécessaire que le film propose un abord des tableaux qu'il serait le premier à promouvoir. Il lui arrive parfois - ce sera le cas ici - de s'inscrire dans un mouvement qui le précède, dont il libelle autrement les problématisations: l'iconologie nostalgique peut tout à fait être une expédition collective. Un mot sur cet adjectif, qui convient mal. Encore une fois - je l'ai dit tout à l'heure rapidement entre parenthèses ; c'est l'occasion d'y revenir - ma démarche ici n'a rien d'auteuriste (résolutions, préméditations, messages), mais ne porte que sur des entre-perceptions d'images les unes par les autres, puisque je n'ai accès qu'aux images. Aussi cette entre-perception n'est pas subordonnée à un corpus d'artiste et peut tout à fait être «poly-auteurale » ou «transauteurale ». Les images qui s'entr'expriment peuvent être apparemment très éloignées dans l'espace, dans le temps, dans la nature médiatique, et ainsi de suite ; une image peut mieux exprimer une image distante qu'une image plus proche (parce qu'elle serait assignée au même artiste, etc.). Seul compte qu'elles s'affectent les unes les autres et forment ainsi un même rang iconique (comme on parle de rang biologique : genres, espèces, familles...). - Ainsi, on trouvera chez Picasso des images ayant déjà souligné la dimension impudique du tableau de Raphaël : plusieurs dessins de la série des 347 gravures, tirées sur vélin par les frères

\footnotetext{
${ }^{33}$ Charles de Montalembert, Mélanges d'art et de littérature, Paris, Jacques Lecoffre et $\mathrm{C}^{\text {ie }}, 1861$, p. 177.

${ }^{34}$ Vasari, Les Vies des meilleurs peintres, sculpteurs et architectes, tome 5, op. cit., p. 222.
} 
Crommelynck dans leur atelier de Mougins, représentent des variations du tableau Raphaël et la Fornarina et retiennent parfaitement la leçon ingresque du regard au et du tiers (maintenant explicite et à voir dans la représentation : Michel-Ange caché sous le tapis ou le pape Léon X assis sur un pot de chambre au vu de tous). La copulation entre Raphaël et la Fornarina est désormais affichée très pornographiquement (pénétration, scatologie).

L'originalité du Rôti de Satan est d'arriver à cette conclusion nostalgique apparemment déconcertante, malséante même, quand on la met en regard de la leggiadria (l'élégance) généralement associée à la peinture de Raphaël, par un autre cheminement dans l'histoire de l'art : les seins pincés. C'est d'autant plus intéressant que j'ai tout à l'heure passé sous silence toute une catégorie de tableaux à seins pincés: les Vierges allaitant leur enfant (Madone au coussin vert de Solario, Saint Luc dessinant la Vierge de van der Weyden). Le thème pourrait sembler ici déplacé, et expliquer ce silence. Il ne l'est pourtant pas tant que cela quand on se souvient que Andrée, qui place sur son sein le sein de la Fornarina, est dans le film justement vierge.

Le Rôti de Satan montre donc à nouveau, mais différemment des Larmes amères de Petra von Kant, principalement par un transplant iconographique pris en charge par le film (une prouesse de personnage), comment la mise en scène, la scénographie des corps et les options esthétiques du cadrage et du montage d'un autre art, en l'occurrence le cinéma, peuvent concourir à inventer un tableau. Les Larmes amères de Petra von Kant ont attiré notre attention, au moins dans Midas devant Bacchus, sur Poussin comme un peintre de nus, par opposition à la doxa de l'artiste pieux (Cambry, Gault de Saint-Germain, ), «spirituel » (Victor Cousin) voire rigoriste (Chennevières-Pointel) ; Le Rôti de Satan, sur Raphaël, ou à tout le moins celui de La Fornarina, comme un peintre égrillard, par opposition à la doxa du peintre de la grâce quasi désincarnée (Quatremère de Quincy, Passavant ${ }^{35}$ ). Dans les deux cas que j'ai examinés, la mise en film de la peinture amène l'œil à revenir sur le tableau de départ pour y cumuler un regard nostalgique, c'est-à-dire initier ou organiser une série du pictural par déplacements médiatiques et inscription dans de nouvelles chaînes d'images à partir de virtualités du tableau de départ. Le destin en est peut-être anachronique mais il faut

\footnotetext{
${ }^{35} \mathrm{Au} \mathrm{XIX}^{\mathrm{e}}$ siècle, Quatremère de Quincy (Histoire de la vie et des ouvrages de Raphaël) et Eugène Müntz (Raphaël. Sa vie, son xuvre et son temps) modifièrent la biographie vasarienne : Raphaël serait mort de surmenage. Pour un point scientifique sur les versions de la mort de Raphaël, cf. D. A. Brown et Konrad Oberhuber, «Monna Vanna and Fornarina : Leonardo and Raphael in Rome », Sergio Bertelli et Gloria Rakamus (éd.), Essays Presented to Myron P. Gilmore, tome II, Florence, La Nouva Italia, coll. « Villa i Tatti », 1978, pp. 59-60.
} 
prendre alors ce terme au sens qu'en donne Jacques Rancière, après Nicole Loraux, celui de ce qui seul fait accès à une économie transversale et un carambolage des immanences : "Une anachronie, c'est un mot, un événement, une séquence signifiante sortis de "leur" temps, doués du même coup de la capacité de définir des aiguillages temporels inédits, d'assurer le saut ou la connexion d'une ligne de temporalité à une autre $^{36}$. »

Walter Benjamin affirme, en une remarque éclairante, que «le médium par lequel les œuvres d'art agissent sur les époques ultérieures est toujours un autre que celui par lequel elles ont agi sur leur époque ${ }^{37} \gg$. La raison principale en est que le médium originel est toujours pour le créateur «si dense autour de son œuvre qu'il ne peut sans doute pas le traverser en se mettant en relation avec l'approche que l'œuvre réclame des hommes ». Il y faut pour cela "le détour d'une relation indirecte », exigeant la prise en charge par un autre médium, plus éloigné techniquement, matériellement et peut-être temporellement, " toujours relativement plus mince ». L'iconologie nostalgique le montre autrement. Comme le revenant derridien, on ne la voit pas venir. Elle pulse d'une présence absente sans soupirs. Elle est à chaque fois le dernier destin de l'œuvre, qui « laisse aussi des souvenirs, qui ne trouvent cependant pas toujours d'héritiers ${ }^{38} »$.

\footnotetext{
36 Jacques Rancière, « Le concept d'anachronisme et la vérité de l'historien », L'Inactuel, $\mathrm{n}^{\circ}$ 6, automne 1996, pp. 67-68. Cf. aussi Georges Didi-Huberman, Devant le temps. Histoire de l'art et anachronisme des images, Paris, Minuit, coll. «Paradoxe », 2000.

37 Walter Benjamin, Fragments, $\mathrm{n}^{\circ}$ 96, traduite de l'allemand par Christophe Jouanlanne et Jean-François Poirier, Paris, PUF, coll. «La Librairie du Collège international de philosophie », 2001, p. 143 (je souligne). Même référence pour les citations suivantes.

${ }^{38}$ W. Benjamin, Euvres III, «Le conteur », traduit de l'allemand par Maurice de Gandillac, Painer Rochlitz et Pierre Rusch, Gallimard, coll. «Folio-Essais », 2006, p. 137.
} 